BENGOUMI (M.), FAYE (B.), EL KASMI (K.), TRESSOL (J.C.). Variability factors in trace elements nutritional status by plasmatic indicators in camel (Camelus dromedarius) in Morocco. I. Normal value and physiological variability. Rev. Élev. Méd. vét. Pays trop., 1995, 48 (3) : 271-276

To study the copper and zinc nutritional status of camel in different ecological areas from Morocco (Guelmim, Laâyoune, Tadla), one-hundredtwenty-five dromedary camels were used in three experiments. The mean

\section{Facteurs de variation des indicateurs plasmatiques du statut nutritionnel en oligo-éléments chez le dromadaire au Maroc II. Effet d'une complémentation minérale}
M. Bengoumi ${ }^{1}$
B. Faye ${ }^{2}$
I.C. Tressol ${ }^{3}$
D. Bengoumi ${ }^{1}$

\begin{abstract}
BENGOUMI (M.), FAYE (B.), TRESSOL (J.C.), BENGOUMI (D.) Facteurs de variation des indicateurs plasmatiques du statut nutritionnel en oligo-éléments chez le dromadaire au Maroc. II. Effet d'une complémentation minérale. Revue Elev. Méd. vét. Pays trop., 1995, 48 (3) : 276-
\end{abstract} 280

Quatre lots de 5 dromadaires ont reçu respectivement une injection unique de cuivre et de zinc (lot IM), une complémentation orale pendant un mois de sulfate de cuivre (lot $\mathrm{Cu}$ ), de sulfate de zinc (lot $\mathrm{Zn}$ ) et aucune complémentation (lot T). L'essai a duré 111 jours et la période de complémentation est survenue entre $7^{\mathrm{e}}$ et le $37^{\mathrm{e}}$ jour. Dans le lot $\mathrm{IM}$, la cuprémie et la zincémie ont significativement augmenté (maximum de 72 et de $73 \mu \mathrm{g} / 100 \mathrm{ml}$ respectivement). Dans le lot $\mathrm{Cu}$, la cuprémie a significativement augmenté ainsi que la zincémie dans le lot $\mathrm{Zn}$, mais les valeurs observées ne dépassent pas les seuils admis comme limite de carence et chutent rapidement après l'arrêt de la complémentation. L'interaction cuivre-zinc se traduit par une dinninution non significative de la zincémie dans le lot $\mathrm{Cu}$ et significative de la cuprémie dans le lot $\mathrm{Zn}$.

Mots-clés : Dromadairc - Complément minćral - Cuivrc - Zinc - Plasma sanguins - Maroc.

1. Laboratoire de Biochimie clinique et nutritionnelle, I.A.V Hassan II, BP 6202, Rabat-Instituts, Maroc.

2. Laboratoire d'Ecopathologie, INRA-Theix, 63122 Saint-GenèsChampanelle, France.

3. Laboratoire des Maladies nutritionnelles, INRA-Theix, 63122 Saint-Genès-Champanelle, France.

Reçu le 26.6.1995, accepté le 20.12.1995. regional plasma values varied from 65 to $102 \mu \mathrm{g} / 100 \mathrm{ml}$ for copper and from 38 to $59 \mu \mathrm{g} / 100 \mathrm{ml}$ for zinc. Except the significant effect of age on plasma zinc concentration which was higher in youngers than adults, there was no significant effect of physiological factors (age and sex) on copper and zinc plasma concentrations. Results are discussed according to the variations of basal diet availability in the different trials.

Key words : Dromedary - Feeding - Copper - Zinc - Blood plasma Morocco.

\section{Introduction}

Des travaux antérieurs réalisés sur le chamelon polycarencé ont montré l'absence d'effet notable d'une complémentation minérale spécifique sur la modification des teneurs plasmatiques en minéraux (4). De même, une complémentation orale de courte durée ne modifie pas la cuprémie et la zincémie des dromadaires recevant une alimentation assurant les besoins d'entretien (2).

Or, la régulation de certains éléments-traces, notamment du zinc, à des niveaux plus bas que chez les autres ruminants domestiques (2) semble faire intervenir non seulement le statut nutritionnel global des animaux (4), mais aussi la voie d'apport de la complémentation et la durée de celle-ci. L'objectif de ce travail est donc d'étudier la relation entre les apports alimentaires en cuivre et en zinc par différentes voies et leurs effets sur les concentrations plasmatiques observées en tenant compte des interférences entre ces éléments-traces.

\section{Matériel et méthodes}

\section{Animaux}

L'étude a été réalisée à la station expérimentale cameline de Laâyoune (Maroc) sur 20 dromadaires d'un poids moyen de $300 \mathrm{~kg}$ environ, âgés de 3 à 4 ans et en bon état de santé. Ces animaux étaient déparasités (injection sous-cutanée d'ivermectine) avant le début de l'expérimentation. Ils ont été maintenus en stabulation entravée et recevaient une ration de base comprenant $2 \mathrm{~kg}$ d'orge concassée, $1 \mathrm{~kg}$ de paille de blé et $1 \mathrm{~kg}$ de foin de luzerne, cette ration correspondant à des besoins d'entretien établis lors de travaux préliminaires (environ $1,2 \mathrm{~kg}$ $\mathrm{MS} / 100 \mathrm{~kg}$ poids vif). Cette ration apportait $34 \mathrm{mg} \mathrm{de}$ cuivre et $62 \mathrm{mg}$ de zinc (analyses effectuées au laboratoire INRA). Les animaux ont été abreuvés ad libitum pendant toute la durée de l'essai. Aucun refus n'a été constaté. 


\section{Dispositif expérimental}

L'expérimentation a comporté, après une phase d'adaptation de 15 jours, les trois étapes suivantes :

1. Etape de contrôle (7 jours) au cours de laquelle les animaux ont été répartis aléatoirement en 4 lots de 5 dromadaires, et ont reçu la même ration de base.

2. Etape de complémentation ( 30 jours) : en plus de la ration de base identique pour tous les animaux, chaque groupe a reçu un des traitements suivants :

- lot IM : administration par une injection intra-musculaire, séparément dans les deux membres postérieurs, de $10 \mathrm{ml}$ de Prolontex-Zinc ${ }^{N D}$ soit $600 \mathrm{mg}$ de Zinc Métal et de $10 \mathrm{ml}$ de Prolontex-Cuivre ${ }^{\mathrm{ND}}$ soit $1600 \mathrm{mg}$ de $\mathrm{Cu}$ Métal au cours du premier jour de cette période ;

- lot $\mathrm{Cu}$ : administration quotidienne par voie orale de $340 \mathrm{mg}$ de sulfate de cuivre pentahydraté dissous dans $200 \mathrm{ml}$ d'eau distribués à la bouteille pendant toute la période de complémentation ;

- lot $\mathrm{Zn}$ : administration quotidienne par voie orale de $2400 \mathrm{mg}$ de sulfate de zinc heptahydraté dissous dans $200 \mathrm{ml}$ d'eau distribués dans les mêmes conditions que précédemment ;

- lot $\mathrm{T}$ : groupe témoin ne recevant aucun traitement.

3. Etape de post-complémentation (75 jours) : pendant cette période, la complémentation des lots $\mathrm{Cu}$ et $\mathrm{Zn}$ a été arrêtée. Cependant les animaux étaient maintenus en lots et recevaient toujours la même ration de base.

\section{Les prélèvements}

Les prélèvements de sang ont été réalisés sur tous les animaux, le matin avant la distribution du repas par ponction de la veine jugulaire sur des tubes vacutainer. Le sérum a été recueilli sur des tubes en plastique et stocké à $-20^{\circ} \mathrm{C}$ jusqu'à l'analyse. Le rythme de prélèvement adopté a été le suivant :

- pendant l'étape contrôle, le sang a été prélevé tous les deux jours, soit 4 prélèvements $(\mathrm{J} 6, \mathrm{~J} 4, \mathrm{~J} 2, \mathrm{~J} 0)$;

- pendant l'étape complémentation, les prélèvements ont été réalisés $6 \mathrm{~h}, 12 \mathrm{~h}, 24 \mathrm{~h}$ et $48 \mathrm{~h}$ après le début de la complémentation, puis une fois tous les deux jours jusqu'à J30, soit au total 18 prélèvements par animal ;

- au cours de la période suivante, les prélèvements ont été réalisés tous les deux jours pendant la première semaine (J32 à J38), puis une fois par semaine durant les trois semaines suivantes (J45, J52, J59), puis une fois par quinze jours jusqu'à la fin de l'essai (J74, J89, J104).

\section{Analyses de sang}

Les concentrations plasmatiques du cuivre et du zinc ont été déterminées par spectrophotométrie d'absorption atomique selon la méthode décrite par Bellanger et Lamand (1).

\section{Traitement statistique}

L'analyse de variance à un critère de variation a été utilisée pour évaluer l'effet des différents traitements sur l'évolution des paramètres plasmatiques étudiés.

\section{Résultats}

Les valeurs moyennes de la cuprémie et de la zincémie observées au cours de l'essai dans les différents lots ont respectivement varié de 41 à $56 \mu \mathrm{g} / 100 \mathrm{ml}$ et de 45 à $53 \mu \mathrm{g} / 100 \mathrm{ml}$ (tabl. l). Les valeurs extrêmes enregistrées ont été de 22 et $81 \mu \mathrm{g} / 100 \mathrm{ml}$ pour le cuivre et de 32 à $80 \mu \mathrm{g} / 100 \mathrm{ml}$ pour le zinc.

\section{TABleaU I}

Valeurs moyennes et écart-types de la cuprémie et de la zincémie calculés sur 5 dromadaires par lot $x 32$ prélèvements, soit 160 prélèvements par groupe

\begin{tabular}{|c|c|c|c|c|}
\hline Parametre & M & cu & $2 \mathrm{n}$ & T. \\
\hline Cuivre $(1 \mathrm{~g} / 100 \mathrm{ml})$ & $56 \pm 8$ & $55+4$ & $41+3$ & $44+2$ \\
\hline Zinc $(\mu g / 100 \mathrm{mil})$ & $53+10$ & $45+2$ & $52+5$ & $46+2$ \\
\hline
\end{tabular}

Aucune différence significative, tant pour le cuivre que pour le zinc n'a été relevée entre les lots pendant la période contrôle ainsi que dans les 5 derniers prélèvements de la période post-complémentation (de J59 à J104).

La cuprémie dans le lot IM a été significativement plus élevée que dans le lot $T$ dès le $2^{\mathrm{e}}$ jour de la complémentation (J2) et le demeure jusqu'à J30 (fig. 1). C'est dans ce lot que la valeur moyenne maximale a été observée $(72 \pm 4 \mu \mathrm{g} / 100 \mathrm{ml})$ à $\mathrm{J} 14$. Dans le lot $\mathrm{Cu}$ (distribution de cuivre per os), la cuprémie est significativement augmentée de $\mathrm{J} 2$ à $\mathrm{J} 36$, soit une période plus longue que le lot précédent. Toutefois, la valeur moyenne maximale enregistrée n'a pas dépassé $60 \mu \mathrm{g} / 100 \mathrm{ml}$ (fig. 1). Bien que faible, la diminution de la cuprémie dans le lot $\mathrm{Zn}$ par rapport au lot témoin est significative $(p<0,01)$ de J16 à J34.

Des évolutions similaires ont été observées pour la zincémie (fig. 2). Dans le lot IM, la différence avec le lot témoin 
cuivre (en $\mu \mathrm{g} / 100 \mathrm{ml}$ )

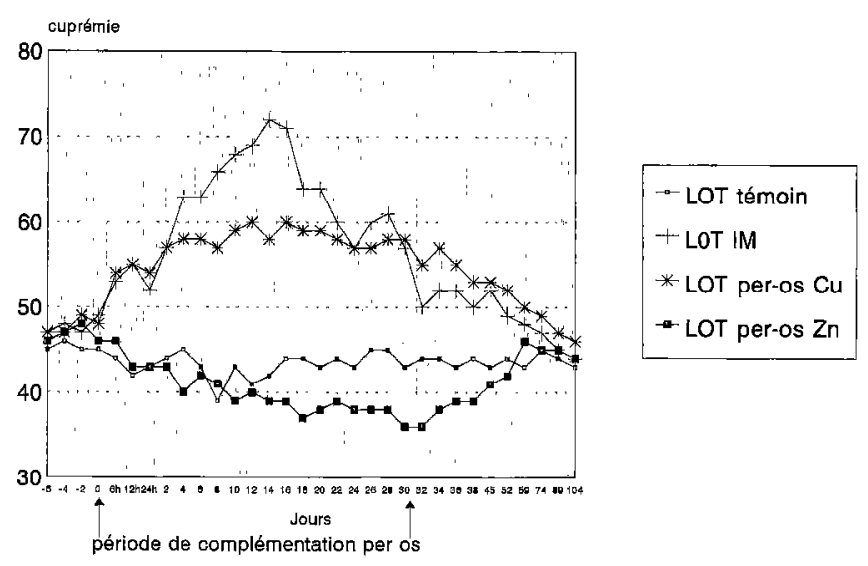

Figure 1 : Evolution de la cuprémie dans les quatre lots de dromadaires.

a été significative entre $\mathrm{J} 1$ et $\mathrm{J} 22$ avec un maximum de $73 \mu \mathrm{g} / 100 \mathrm{ml}$ à J14. Dans le lot Zn, l'augmentation de la zincémie était significative à partir de $\mathrm{J} 4$ et jusqu'à $\mathrm{J} 32$ avec un maximum observé de $59 \mu \mathrm{g} / 100 \mathrm{ml}$ à J12. La légère diminution de la zincémie observée entre $\mathrm{J} 10$ et J30 dans le lot $\mathrm{Cu}$ par rapport au lot témoin n'est pas significative (fig. 2).

\section{Discussion}

La ration de base distribuée aux dromadaires apportait environ $10 \mathrm{mg}$ de cuivre et $17 \mathrm{mg}$ de zinc par $\mathrm{kg}$ de MS selon les analyses effectuées à l'INRA, soit des quantités juste suffisantes pour le cuivre et très inférieures aux besoins pour le zinc d'après les recommandations préconisées pour cette espèce (4). II apparaît en effet que les besoins du dromadaire en éléments-traces rapportés au $\mathrm{kg}$ de MS ingérée sont plus élevés que chez les bovins. Cependant, compte tenu de la plus faible quantité de MS ingérée par cette espèce comparativement à des bovins de même poids (environ deux fois moins), et de la faible qualité nutritionnelle globale des rations généralement à la disposition de ces animaux, les quantités recommandées exprimées en $\mathrm{mg}$ par $\mathrm{kg}$ de poids vif pour le dromadaire demeurent comparables à celles des bovins.

\section{Cuivre}

En début d'essai, après 15 jours d'adaptation, les animaux présentaient des valeurs moyennes de cuprémie inférieures à la limite de carence généralement admise pour les ruminants $(60 \mu \mathrm{g} / 100 \mathrm{ml})$. L'injection de cuivre a modifié los concentrations sériques en cet élément dès le $2^{\mathrm{e}}$ jour suivant le traitement et a permis d'atteindre la

\section{zinc (en $\mu \mathrm{g} / 100 \mathrm{ml})$}

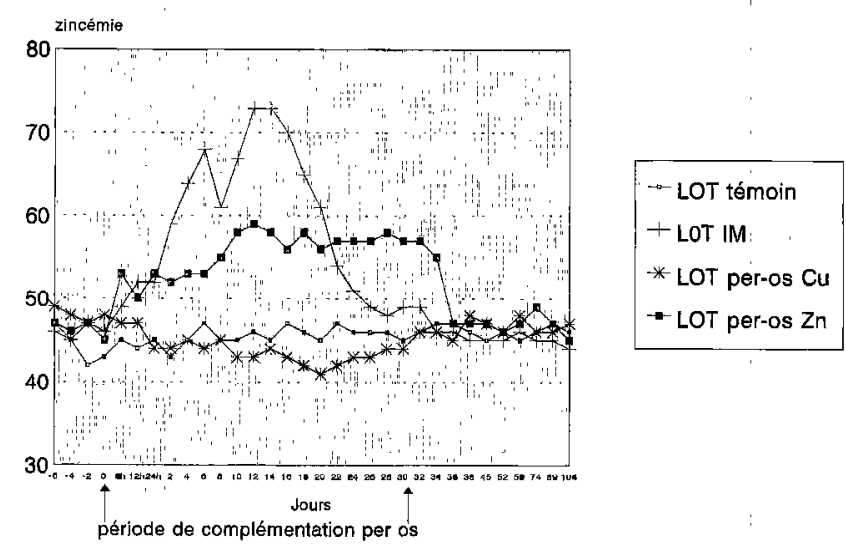

Figure 2 : Evolution de la zincémie dans les quatre lots de dromadaires.

valeur-seuil de $60 \mu \mathrm{g} / 100 \mathrm{ml}$ dès le $4^{\mathrm{e}}$ jour. Le maintien de la cuprémie au-dessus de ce seuil a duré près d'un mois, soit une durée inférieure à celle obtenue chez des ovins en Ethiopie (3) dans des situations de carence comparables, mais similaire à celle observée en France sur des moutons non-carencés (8).

Conformément aux observations faites sur le mouton (9), aucune réaction inflammatoire locale n'a été décelée au point d'injection.

L'apport par voie orale de $340 \mathrm{mg}$ de sulfate de cuivre a permis d'augmenter la quantité de cuivre ingérée, la faisant passer de $10 \mathrm{mg}$ à $33 \mathrm{mg} / \mathrm{kg} \mathrm{MS} / \mathrm{j}$, soit un peu plus du double des apports recommandés par Faye et al. (4) pour le dromadaire. Cette forme de complémentation a permis une augmentation significative de la cuprémie dans le lot $\mathrm{Cu}$ dès le second jour, mais celle-ci ne s'est maintenue qu'une semaine après l'arrêt de la complémentation. Ce dernier résultat est similaire à celui observé à Djibouti sur des chamelons carencés ayant reçu une complémentation orale pendant 3 mois (4). Par ailleurs, les concentrations maximales atteintes $(60 \mu \mathrm{g} / 100 \mathrm{ml})$ demeurent à la limite inférieure des valeurs observées au Maroc chez le dromadaire sur parcours (1). On peut considérer que les animaux dans notre essai étaient en situation de déficit cuprique et que l'apport de $33 \mathrm{mg}$ de cuivre par $\mathrm{kg} / \mathrm{MS}$ a permis d'élever les concentrations plasmatiques sans favoriser un stockage hépatique. Ces observations confirment celles faites par Faye et al. (4) qui ont recommandé chez le dromadaire en croissance, des apports quotidiens de l'ordre de 15 à $20 \mathrm{mg}$ de cuivre $/ \mathrm{kg} \mathrm{MS}$, soit des valeurs sensiblement plus élevées que chez les bovins (10).

Dans le lot $\mathrm{Zn}$, la diminution sensible de la cuprémie peut être attribuée à une interaction négative mutuelle entre le 
cuivre et le zinc durant le processus d'absorption au niveau de la muqueuse intestinale, phénomène déjà mis en évidence chez le rat $(16,17)$. Chez les bovins, une ration très enrichie en zinc diminue la concentration en cuivre plasmatique jusqu'à des niveaux de carence (15). Cette interaction est due à une compétition dans le transport des éléments-traces au niveau des sites d'absorption de la paroi intestinale (13).

\section{Zinc}

La zincémie moyenne relevée pendant la phase de contrôle était dans l'intervalle des valeurs usuelles du dromadaire (5). L'injection de zinc a entraîné une augmentation nette de la zincémie dès le premier jour et pendant 3 semaines, soit une durée sensiblement plus courte que chez le mouton (12). Cependant, le niveau maximum atteint de la zincémie $(73 \mu \mathrm{g} / 100 \mathrm{ml})$ reste relativement faible par rapport aux résultats rapportés par d'autres espèces de ruminants (8).

La distribution per os de $2400 \mathrm{mg}$ de sulfate de zinc a permis de multiplier par 10 la quantité ingérée de zinc (de 18 à $185 \mathrm{mg} / \mathrm{kg} \mathrm{MS}$ ). Cette quantité représente à peine le double des apports recommandés par Faye et al. (4) chez le dromadaire $(100 \mathrm{mg} / \mathrm{kg} \mathrm{MS})$, et plus de trois fois ceux recommandés chez les bovins (11).

La complémentation orale en zinc a permis une amélioration notable de la zincémie, mais celle-ci demeure inférieure à $60 \mu \mathrm{g} / 100 \mathrm{ml}$ et chute très rapidement dès la fin de la complémentation, conformément aux résultats déjà obtenus à Djibouti sur des chamelons carencés et complémentés pendant 3 mois (4). L'évolution en plateau de la zincémie et la chute brutale après l'arrêt de la complémentation s'explique par l'absence de forme connue de stockage du zinc dans les tissus des animaux domestiques (14). II est connu par ailleurs que, aussi bien chez la brebis (10) que chez le chamelon (4), la complémentation orale en zinc s'avère inefficace lorsque la ration de base est pauvre en azote.

L'interaction cuivre-zinc dans le lot $\mathrm{Cu}$ est moins marquée que dans le lot $\mathrm{Zn}$. Ceci peut être attribué à une régulation de la zincémie sensiblement différente chez le dromadaire, ce que confirment les résultats récents de la littérature concernant les niveaux usuels de la zincémie dans cette espèce $(6,7)$.

\section{Conclusion}

Ces résultats montrent qu'il subsiste encore des zones d'ombre dans la compréhension de la régulation des éléments-traces tels que le cuivre et le zinc chez le dromadaire. II conviendrait à l'avenir de prendre en considération des périodes de complémentation plus longues et, surtout, d'estimer le rôle du stockage hépatique et de l'excrétion urinaire et fécale dans les mécanismes de régulation des minéraux concernés. Une étude comparative bovins-camelins est mise actuellement en place dans ce sens. La comparaison avec les bovins permettra, en outre, de déterminer la spécificité physiologique du dromadaire, en relation avec ses capacités d'adaptation aux conditions désertiques.

\section{Remerciements}

Nous tenons à remercier la Fondation internationale pour la science (IFS) pour son appui financier dans la réalisation de ce travail (Bourse B/2293 - 1). Les auteurs remercient également le Docteur Hidane Kamal, Chef des Services vétérinaires de Laâyoune pour son aide.

\section{Bibliographie}

1. BELLANGER J., LAMAND M., 1975. Méthode de dosage du cuivre et du zinc plasmatique. Bull. tech. CRZV Theix, INRA, 20 : 23-54.

2. BENGOUMI M., FAYE B., EL KASMI K., TRESSOL J.C., 1995. Facteurs de variation des indicateurs plasmatiques du statut nutritionnel en oligo-éléments chez le dromadaire au Maroc. I. Valeurs usuelles et variations physiologiques. Rev. Élev. Méd. vét. Pays trop., 48 : 271-276.

3. FAYE B., GRILLET C., 1984. La carence en cuivre chez les ruminants domestiques dans la région d'Awash (Ethiopie). Rev. Élev. Méd. vét. Pays trop., 37 (1) : 42-60.

4. FAYE B., SAINT-MARTIN G., CHERRIER R., ALI RUFFA, 1992. The influence of high dietary protein, energy and mineral intake on deficient young camels in Djibouti. II. Changes in mineral status. Comp. Biochem. Physiol., 102A : 417-424.

5. FAYE B., BENGOUMI M., 1994. Trace-elements status in camels. A review. Biol. trace Elem. Res., 41 : 1-11.

6. FAYE B.. JOUANY J., CHACORNAC J.P., RATOVONANAHARY M., 1995. L'élevage des grands camélidés. Analyse des initiatives réalisées en France. INRA Prod, anim.. 8 (1) : 3-17.

7. FAYE B., CHACORNAC J.P.. RATOVONANAHARY M., SOUBRE P., 1995. Metabolic profiles and health status of camel in temperate conditions. Comp. Biochem. Physiol., 112A (1) : 67-73.

8. LAMAND M., 1978. Copper and zinc deficiencies treatment by intramuscular injections in sheep. Ann. Rech. vét., 9 (3) : 495-500.

9. LAMAND M., 1978. Copper deficiency prophylaxis in grazing sheep by copper oxide injection. Am. Rech. vét., 9 (3) : 501-504.

10. LAMAND M. 1985. Influence of protein intake on per os deficiency treatment in sheep. Am. Rech. vét., 16 (3) : 285-287.

11. LAMAND M., 1987. Les besoins en oligo-éléments des ruminants. Bull. tech. CRZV Theix, INRA, 70:113-116. 


\section{Communication}

12. LAMAND M., LAB C., TRESSOL J.C., 1980. Cumparaison of the efficiency of zinc injected as metal oxide for zinc deficiency treatment in sheep. Ann. Rech. vét., 11 (2) : 147-149.

13. Mc DOWELL L.R., 1992. Minerals in animal and human nutrition. San Diego, USA, Academic Press, 517 p.

14. Mc DOWELL L.R., CONRAD J.M., GLEN HEMBRY F., 1993. Mineral grazing ruminants in tropical regions, $2^{\text {nd }}$ Ed., USA, U.S.A.I.D. and C.B.A.G., Univ. Florida, 65 p.

15. TOWERS N.R., YOUNG P.W., WRIGHT D.E., 1981. Effect of zinc supplementation on bovine plasma copper. N.Z. vet. J., $29: 113114$.

16. VAN CAMPEN P.R., 1971. Copper inference with intestine absorption of zinc ${ }^{65}$ rats. J. Nutr, $97: 104$.

17. YU S., BEYNEN A.C., 1994. The combined effect of high iron and zinc intake on copper status in rats. Biol. trace Elem. Res., 42 : 71-79.
BENGOUMI (M.), FAYE (B.), TRESSOL (J.C.), BENGOUMI (D.). Variability factors in trace elements nutritional status by plasmatic indicators in camel (Camelus dromadarius) in Morocco. II. Effect of mineral supplement. Revae Élev. Méd. vél. Pays' lrop., 1995, 48 (3) :276-280.

Four groups of 5 camels received one injection of copper and zinc (IM group), an oral supplementation during one month with copper sulphate ( $\mathrm{Cu}$ group), or zinc sulphate ( $\mathrm{Zn}$ group), and no supplementation ( $T$ group) respectively. The trial was performed during 111 days and complementation occurred from day 7 to 37 . In the IM group, cupremia and zincemia significantly increased $(72$ and $73 \mu \mathrm{g} / 100 \mathrm{ml}$ maximum respectively). Cupremia increased in Cu group as zincemia in $\mathrm{Zn}$ group but the observed values did not overpass the threshold of admitted deficiency limit, and fall down fastly after the end of the supplementation period. The interaction copper-zinc explained the non-significant decreasing of zincemia in $\mathrm{Cu}$ group and significant decreasing of cupremia in $\mathrm{Zn}$ group.

Key words : Dromedary - Mineral supplement - Copper - Zinc - Blood plasma - Morocco. 